\title{
TENDENCIAS GLOBALES EN EL CONTROL DE LA CONTAMINACIÓN VEHICULAR SITUACIÓN A 2008*
}

\author{
Michael P. Walsh
}

\begin{abstract}
Desde fines de la Segunda Guerra Mundial ha habido un crecimiento fuerte y sostenido del parque vehicular. Este aumento se ha convertido en una fuente importante, si no dominante, de contaminación atmosférica. Este trabajo revisa los patrones históricos de crecimiento del parque automotriz y sus consecuencias ambientales, como también los esfuerzos en materia de control de emisiones a nivel mundial y las nuevas iniciativas por transformar los vehículos y los combustibles para minimizar efectos ambientales.
\end{abstract}

Se presentan los avances logrados a la fecha en la reducción de emisiones para vehículos a combustibles diésel y gasolina, concluyéndose

Michael P. Walsh. Ingeniero mecánico, especialista en control de la contaminación vehicular. Durante varios años se desempeñó como director del área de control de contaminación de vehículos motorizados de la Ciudad de Nueva York y en la EPA (US Environmental Protection Agency). Actualmente codirige el Subcomité Técnico Asesor de Fuentes Móviles de la EPA y se desempeña como consultor independiente asesorando a gobiernos e industrias a nivel mundial. Ha participado en programas de transporte y manejo de calidad del aire en México, China, Brasil, Hong Kong, Filipinas y Vietnam. Entre otras distinciones recibidas, la EPA le otorgó la primera versión del premio "Lifetime Individual Achievement Award” por su carrera y aportes a un aire limpio. Públicos.

* Trabajo presentado el 4 de diciembre de 2008 en el Centro de Estudios

Traducción al castellano de María José Morandé para Estudios Públicos.

Estudios Públicos, 114 (otoño 2009). 
que una de las lecciones más importantes aprendidas en los cerca de cincuenta años de historia del control de la contaminación vehicular a nivel mundial es que los vehículos y los combustibles deben tratarse como un sistema. Las mejoras en los vehículos y los combustibles deben ir de la mano si se espera avanzar en la disminución de la contaminación. Un programa focalizado únicamente en los vehículos está condenado al fracaso; de igual manera, tampoco tendrá éxito un programa diseñado para mejorar solamente la calidad del combustible. Luego se discuten las distintas vertientes normativas sobre emisiones vehiculares con énfasis en los tres programas mundiales predominantes, que corresponden a EE.UU. (incluida California), la Unión Europea (UE) y Japón. Por último, se analizan aspectos relacionados con el cambio climático global, estrategias para reducir los gases de efecto invernadero y la contaminación del aire, y tecnologías vehiculares avanzadas, tales como vehículos eléctrico a batería de alto rendimiento, vehículos eléctricos de ciudad, vehículos eléctricos de vecindario, vehículos eléctricos híbridos, vehículos eléctricos híbridos enchufables y vehículos eléctricos con celda de combustible.

Palabras clave: Contaminación vehicular, normas de emisiones vehiculares; emisiones vehiculares; nuevas tecnologías vehiculares; combustibles alternativos.

\section{Introducción}

$\mathrm{D}$ esde fines de la Segunda Guerra Mundial ha habido un crecimiento fuerte y sostenido del parque vehicular. Al comienzo se concentró principalmente en Norteamérica, pero en las últimas seis décadas éste se ha propagado gradualmente, primero en Europa, ahora en Asia y, en menor grado, en Latinoamérica. Es evidente que los vehículos han mejorado mucho la calidad de vida de las personas; han aportado mayor movilidad, trabajo, oportunidades recreacionales, por nombrar sólo algunos aspectos. Sin embargo, también han transformado muchas ciudades en conurbaciones descontroladas, han generado a la fecha una insaciable sed por un preciado y limitado petróleo, y se han convertido en una fuente importante, si no dominante, de contaminación atmosférica urbana y, en el último tiempo, en la fuente que aumenta más rápido entre todas las que contribuyen al cambio climático.

El propósito de este trabajo es revisar estos antecedentes, centrándose primero en los patrones históricos de crecimiento del parque 
automotor y en las consecuencias ambientales resultantes; luego, en los actuales esfuerzos de control a nivel mundial y, por último, en las nuevas iniciativas por transformar los vehículos y los combustibles con el fin de permitir un mayor uso vehicular y, al mismo tiempo, minimizar los efectos ambientales.

\section{Tendencias en la producción vehicular mundial}

El crecimiento total de la producción de vehículos a motor, especialmente desde fines de la Segunda Guerra Mundial, ha sido impresionante, aumentando desde unos cinco millones de unidades al año hasta más de sesenta millones. Tal como se aprecia en el Gráfico $\mathrm{N}^{\circ} 1$, entre 1970 y 2005, aproximadamente un millón adicional de vehículos se ha fabricado al año, en comparación con el año anterior, alcanzándose una producción de casi sesenta y seis millones de vehículos en 2005ํ․ Los datos acerca de la producción de motocicletas son menos precisos, pero un importante fabricante ${ }^{2}$ estima que la producción global superó los treinta millones de unidades en 2003 y está aumentando en un millón de unidades al año.

\section{GRÁFICO N 1: $\quad$ PRODUCCIÓN ANUAL DE AUTOMÓVILES, CAMIONES Y BUSES}

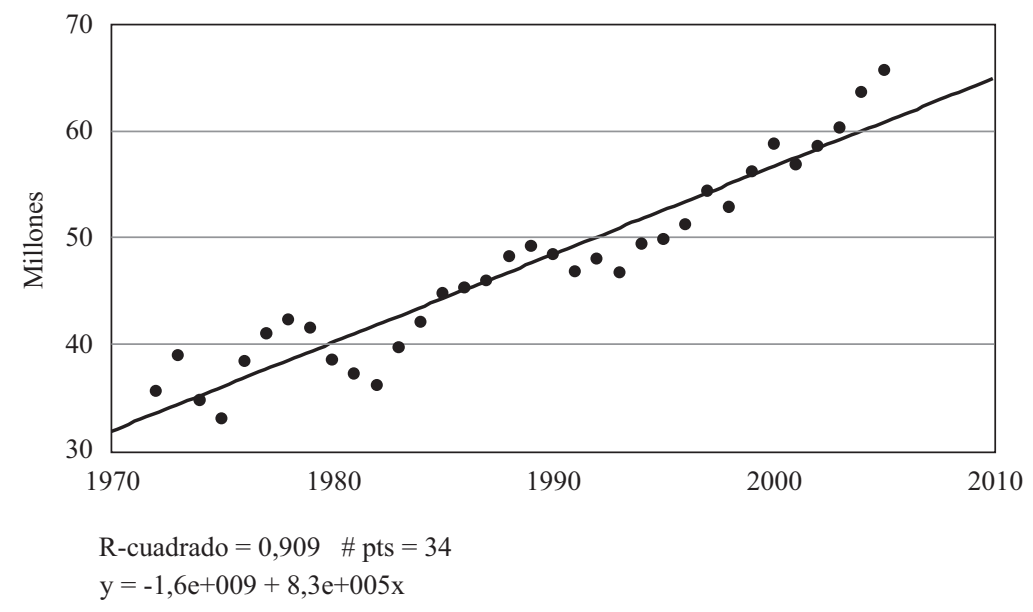

Fuente: Elaboración propia del autor.

${ }^{1}$ Wards Communications Inc.: Ward's Motor Vehicle Data, 2006.

${ }^{2}$ Honda Motor Company: “World Motorcycle Facts and Figures”, 2004. 


\section{Tendencias mundiales de los parques vehículares}

Debido al continuo crecimiento de la población, así como de las áreas urbanas y de las expectativas de mejoría económica, se puede prever un aumento sostenido y sustancial en el parque vehicular global ${ }^{3}$ de acuerdo con las tendencias históricas ilustradas en el Gráfico $N^{\circ} 2$. El parque vehicular global superó los mil millones de unidades en 2002 y ha continuado en sostenida alza desde entonces.

\section{GRÁFICO Nº 2: $\quad$ PRODUCCIÓN MUNDIAL DE VEHÍCULOS A MOTOR}

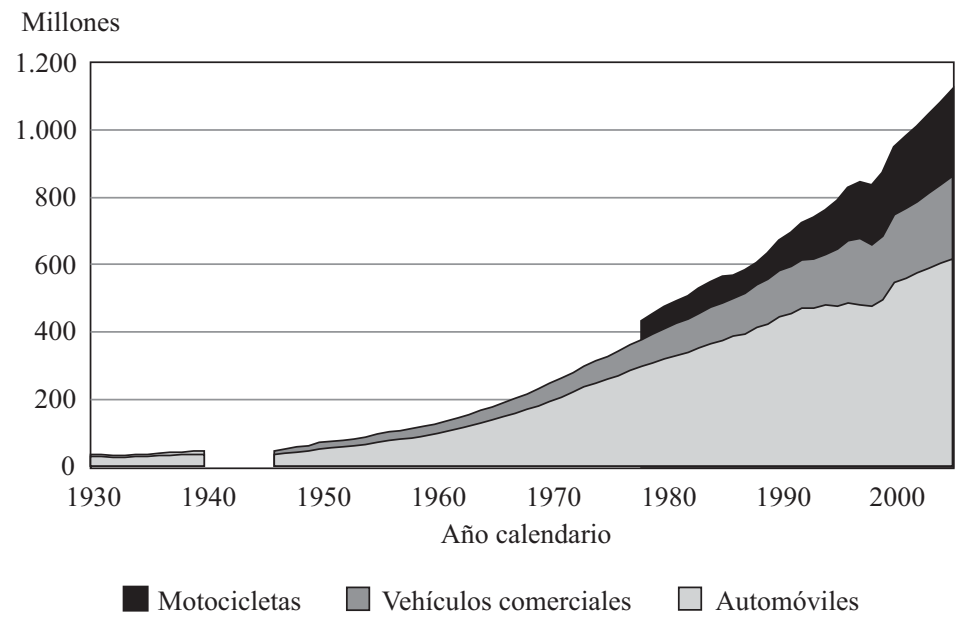

Fuente: Elaboración propia del autor.

\section{Tendencias en las emisiones de los vehículos a motor}

Los vehículos a motor emiten grandes cantidades de monóxido de carbono (CO), hidrocarburos (HC), óxidos de nitrógeno (NOx), óxidos de azufre (SOx) y sustancias tóxicas tales como benceno, formaldehído, acetaldehído, 1,3-butadieno, partículas finas (MP10 y MP2,5) y (donde aún se añade plomo a la gasolina) plomo. Cada uno de éstos, junto con contaminantes secundarios, tales como el ozono y

${ }^{3}$ World Business Council on Sustainable Development: "Mobility 2030: Meeting the Challenges to Sustainability”, 2004. 
partículas pequeñas (nitratos y sulfatos), pueden ser muy dañinos para la salud y el ambiente. En vista de los crecientes parques automotores y sus resultantes emisiones, la fracción de la contaminación originada por los vehículos que es perjudicial para la salud sigue siendo importante en el mundo desarrollado y está aumentando en muchas ciudades del mundo en desarrollo.

Los gases de efecto invernadero (GEI) que se identifican más con el sector transporte son los principales gases considerados en el Protocolo de Kioto: $\mathrm{CO}_{2}$, óxido nitroso y metano. El potencial de calentamiento global (PCG) del óxido nitroso y metano, en relación con el $\mathrm{CO}_{2}$, aparece en el Cuadro $\mathrm{N}^{0}$ 1. Sin embargo, es importante señalar que otros contaminantes relacionados con los vehículos también contribuyen al calentamiento global, aunque su cuantificación ha sido más difícil; éstos incluyen CO, HCNM y dióxido de nitrógeno. Hay consenso general, por ejemplo, en que el $\mathrm{CO}$ que emiten los vehículos se convierte eventualmente en dióxido de carbono en la atmósfera, y en el proceso consume radicales hidroxilos que por otra parte podrían reducir las concentraciones de metano. De manera similar, los HCNM y los NOx contribuyen a la masa global de ozono troposférico, un poderoso gas de efecto invernadero. Los PCG enumerados en el Cuadro $\mathrm{N}^{\circ} 1$, incluidos aquellos atribuidos a CO, HCNM y dióxido de nitrógeno, corresponden al informe original (1990) del Panel Intergubernamental sobre Cambio Climático (IPCC, por su sigla en inglés) ${ }^{4}$.

CUADRO N ${ }^{\circ}$ 1: POTENCIAL DE CALENTAMIENTO GLOBAL DE LAS EMISIONES CONTAMINANTES DEL TRANSPORTE

\begin{tabular}{|c|c|c|c|c|c|}
\hline PCG & $\begin{array}{l}\text { Dióxido } \\
\text { de carbono } \\
\left(\mathrm{CO}_{2}\right)\end{array}$ & $\begin{array}{c}\text { Metano } \\
\left(\mathrm{CH}_{4}\right)\end{array}$ & $\begin{array}{l}\text { Óxido } \\
\text { nitroso } \\
\left(\mathrm{N}_{2} \mathrm{O}\right)\end{array}$ & $\begin{array}{l}\text { Monóxido } \\
\text { de carbono } \\
\text { (CO) }\end{array}$ & $\begin{array}{cc}\text { Hidrocarburos } & \text { Dióxido } \\
\text { no-metano } & \text { de nitrógeno } \\
(\mathrm{HCNM}) & \left(\mathrm{NO}_{2}\right)\end{array}$ \\
\hline
\end{tabular}

Horizonte de de cien años 1 21 310 3 11 7

Fuente: Panel Intergubernamental sobre Cambio Climático, 1990 y 1996.

\footnotetext{
${ }^{4}$ Dada la dificultad para llegar a acuerdo sobre la cuantificación apropiada, los índices PCG específicos para estos gases no están contenidos en el informe más reciente del Panel Intergubernamental sobre Cambio Climático.
} 
Existe cada vez más preocupación de que el carbono negro (CN) u hollín que emiten los vehículos diésel y otras fuentes sea un poderoso gas de efecto invernadero, en parte debido al efecto del albedo de la nieve (reflectividad) en la radiación solar. De acuerdo a lo señalado recientemente por Ramanathan y Carmichael (2008), “[d]ebido a la combinación de una elevada absorción, una distribución regional aproximadamente en línea con la radiación solar y la capacidad para formar, en una mezcla con otros aerosoles, extensas nubes marrones atmosféricas, las emisiones de carbono negro constituyen el segundo elemento que más contribuye al calentamiento global actual. En la región de los Himalaya, el calentamiento solar como consecuencia del carbono negro a grandes alturas puede ser casi tan importante como el dióxido de carbono en el derretimiento de acumulaciones de nieve y glaciares" 5 .

En los principales países industrializados ha habido grandes avances en la reducción de emisiones de los contaminantes atmosféricos urbanos y de sus precursores, los automóviles gasolineros, y están empezando a incorporarse rigurosas exigencias para los vehículos diésel. Sin embargo, se espera que el parque automotor y los kilómetros viajados sigan aumentando rápidamente en el futuro, en particular en los países en desarrollo, lo cual contrarrestará muchos de los logros obtenidos a la fecha ${ }^{6}$.

\section{Avances logrados a la fecha en la reducción de emisiones}

Casi en cualquier rincón del mundo, para cada tipo de vehículo y su correspondiente combustible hay una clara tendencia hacia exigencias cada vez más rigurosas sobre emisiones. Durante la próxima década, este patrón de control será similar para los vehículos todoterreno y sus combustibles. Varios factores impulsan estas tendencias:

- Crecimiento continuo de la cantidad de vehículos y su concentración en áreas urbanas donde los niveles de contaminación siguen siendo inaceptablemente elevados;

\footnotetext{
${ }^{5}$ Ramanathan, V. y G. Carmichael: “Global and Regional Climate Changes Due to Black Carbon”, 2008.

${ }^{6}$ World Business Council on Sustainable Development: "Mobility 2030: Meeting the Challenges to Sustainability”, 2004.
} 
- creciente acumulación de estudios de salud que muestran efectos adversos a niveles más y más bajos de contaminación, y en el caso de MP, virtualmente a cualquier nivel; $y$

- avances en la tecnología vehicular y combustibles limpios, lo cual está haciendo posible lograr niveles de emisión cada vez más bajos a costos razonables.

Una de las lecciones cruciales aprendidas hasta la fecha es que los vehículos limpios y los combustibles de alta calidad van de la mano, por lo que deben tratarse como un sistema. La siguiente sección analizará el efecto del combustible en las emisiones y el progreso alcanzado en el mejoramiento de la calidad de los combustibles y las tecnologías vehiculares.

A lo largo de aproximadamente los últimos veinte años se han realizado exhaustivos estudios para establecer mejor los nexos entre combustibles, vehículos y emisiones vehiculares. Un estudio importante, el programa Auto/Oil Air Quality Improvement Research Program (AQIRP) se inició en 1989 en EE.UU. e involucró a catorce petroleras, tres fabricantes nacionales y cuatro miembros asociados ${ }^{7}$. En 1992, la Comisión Europea también emprendió un programa sobre emisiones vehiculares y calidad del aire. Se invitó a la industria automotriz (representada por la Association des Constructeurs Européens d'Automobiles [ACEA]) y a la industria petrolera (European Petroleum Industry Association [EUROPIA]) a cooperar en un programa marco, que más tarde se conoció como "la actividad tripartita” o European Auto/OIL Program. En junio de 1993, ambas industrias firmaron un contrato para emprender un programa común de prueba, llamado Programa Europeo sobre Emisiones, Carburantes y Tecnologías de Motores (EPEFE, por su sigla en inglés).

El Programa Japonés de Aire Limpio (JCAP, por su sigla en inglés) fue llevado a cabo por el Centro de Energía del Petróleo como un plan de investigación conjunta de la industria automotriz (como usuaria de combustible) y la industria petrolera (como fabricante de combustible), con el apoyo del Ministerio de Economía, Comercio e Industria. El programa contemplaba dos etapas: la primera, llamada JCAP I, comenzó el año 1997 y continuó hasta el año 2001; la segunda, llamada JCAP II, comenzó el año 2002 y continuó hasta el año 2007 con el fin de profun-

\footnotetext{
${ }^{7}$ Auto/Oil Air Quality Improvement Research Program: “Final Report”, 1997.
} 
dizar las actividades de investigación de JCAP I. En JCAP II los estudios se focalizaron en el automóvil y las tecnologías de combustible del futuro con el objeto de reducir a cero las emisiones y, al mismo tiempo, disminuir el consumo de combustibles.

\section{a) Vehículos y combustibles diésel}

Los motores diésel emiten más óxidos de nitrógeno (NOx) y material particulado (MP) que los motores gasolineros equivalentes. Reducir las emisiones de MP tiende a ser la principal prioridad porque los niveles de MP en el ambiente generalmente superan los niveles recomendados por la OMS y son responsables de cientos de miles de muertes prematuras al año. Las materias particuladas de diésel (hollín) han sido consideradas particularmente peligrosas, y tanto el California Air Resources Board como otros organismos las han caracterizado como tóxicas. Las emisiones de NOx, sin embargo, también son importantes, puesto que causan o contribuyen al dióxido de nitrógeno, al ozono y al MP secundario (nitratos) presentes en el ambiente ${ }^{8}$.

Puede lograrse un control moderado a significativo de NOx en los motores diésel por la vía de retardar el tiempo de inyección de combustible e incorporar el sistema EGR (recirculación de gases de escape). También puede sincronizarse la inyección computarizada de combustible a alta presión para reducir las emisiones de MP. Modificar los parámetros del motor para reducir simultáneamente tanto los NOx como el MP es difícil, amén de tener muchas restricciones, puesto que las configuraciones óptimas para un contaminante frecuentemente aumentan las emisiones del otro. Por consiguiente, para alcanzar niveles muy bajos de NOx y MP, es necesario tratar los gases de escape. Los adsorbentes de NOx, la reducción catalítica selectiva, las trampas de almacenamiento de NOx con reducción periódica, los filtros de partículas con regeneración periódica y los catalizadores de oxidación con regeneración continua son tecnologías que se están introduciendo en diferentes niveles en varias partes del mundo. A nivel mundial, por

${ }^{8}$ Ciertos contaminantes que los vehículos emiten como gases sufren una transformación en la atmósfera y se convierten en partículas. Por ejemplo, algunos de los óxidos de nitrógeno gaseosos (NOx) emitidos por los vehículos reaccionan con otros gases y se convierten en nitratos, lo que contribuye a los niveles de material particulado del aire urbano. 
ejemplo, Japón tiende a liderar en el uso extendido de filtros de partículas en los vehículos diésel nuevos, en tanto que Europa se está quedando atrás ${ }^{9}$. Un nuevo tipo de diésel, el motor HCCI (de carga homogénea y encendido por compresión), que presenta otro enfoque para reducir los NOx y las partículas, está recibiendo mucha atención y dentro de unos pocos años podría incorporarse en algunos motores.

El combustible diésel es una mezcla compleja de hidrocarburos cuyos grupos principales son las parafinas, los naftenos y los aromáticos. El azufre orgánico también es un componente natural. Generalmente se emplean aditivos para influir en propiedades tales como las características de flujo, almacenamiento y combustión del combustible diésel. Las propiedades reales del diésel comercial para vehículos dependen de las prácticas de refinación empleadas y de la naturaleza de los petróleos crudos a partir de los cuales se produce el combustible. La calidad y la composición del combustible diésel pueden influir significativamente en las emisiones de los motores diésel.

Para reducir las emisiones de MP y NOx de un motor diésel se debe considerar que la característica más importante del combustible es el azufre, el cual contribuye directamente a las emisiones de partículas y los altos niveles del mismo impiden el uso o perjudican el rendimiento de las tecnologías más eficaces de control de MP y NOx. Para el control de MP, la mayoría de los vehículos nuevos en Japón y los EE.UU., así como en una parte cada vez más significativa en Europa, están equipados con filtros o trampas que reducen sobre el $90 \%$ de las partículas. Se están comenzando a introducir los sistemas de adsorbentes de NOx y la reducción catalítica selectiva; los adsorbentes de NOx son especialmente sensibles a los niveles de azufre en el combustible.

El azufre es un componente natural del petróleo crudo, y el contenido de azufre en el combustible diésel depende tanto de la fuente de petróleo crudo como del proceso de refinación.

La contribución del azufre del combustible diésel a la emisión de partículas en los gases de escape ha quedado demostrada mediante una relación lineal general entre los niveles de azufre y esta emisión regulada. También hay una relación indirecta, ya que algunas emisiones de

${ }^{9}$ Algunos países europeos están usando incentivos tributarios para acelerar la introducción de filtros de MP, de tal modo de alcanzar niveles por encima de la tasa requerida por los nuevos estándares vehiculares euro. 
dióxido de azufre eventualmente se convertirán en la atmósfera en MP sulfatado $^{10}$.

Los motores diésel livianos $(<3,5$ toneladas de peso bruto del vehículo $[\mathrm{PBV}])$ generalmente requieren catalizadores de oxidación para dar cumplimiento a los estándares Euro II o normas de emisiones vehiculares más exigentes. Los catalizadores de oxidación reducen las emisiones de hidrocarburos, monóxido de carbono y partículas, por lo general eliminando el 30\% de la masa total de emisiones particuladas a través de la oxidación de una gran proporción de la parte orgánica soluble. La conversión de azufre en el catalizador reduce la disponibilidad de sitios activos en la superficie del catalizador y, por ende, reduce la eficacia del mismo. Esta desactivación del catalizador es reversible mediante la exposición a alta temperatura — los compuestos de azufre se descomponen y son liberados del recubrimiento del catalizador. Sin embargo, generalmente debido a bajas temperaturas de los gases de escape, en muchas aplicaciones de los motores diésel rara vez se dan las condiciones necesarias para una completa regeneración. Un contenido elevado de azufre en el combustible también puede provocar la formación de sulfatos en el convertidor, los cuales se emiten luego como más partículas. Por consiguiente, es importante hacer coincidir los niveles de azufre con la tecnología post-tratamiento presente en la flota vehicular.

Para que sea posible cumplir con los estándares más exigentes de emisiones de partículas y NOx en los vehículos diésel, en muchos países se están estableciendo límites más restrictivos para el contenido máximo de azufre en el combustible. Si bien pueden lograrse disminuciones importantes en las emisiones de partículas sin reducir los niveles de azufre, generalmente no es posible dar cumplimiento a los estándares Euro II o normas más exigentes de emisiones cuando los niveles de azufre en el combustible superan las 500 ppm, dada la proporción relativamente mayor de sulfatos en la masa total de emisiones de partículas.

En el caso de los estándares Euro III y Euro IV sobre emisiones vehiculares, se requerirán niveles de azufre (350 ppm y 50 ppm, respectivamente) incluso menores para asegurar el cumplimiento de las normas. Para ajustarse a los estándares Euro V y Euro VI o los están-

10 Similar a la transformación secundaria de NOx en nitrato mencionada anteriormente. 
dares norteamericanos Tier 2, los niveles máximos de azufre deberán ser tan bajos como 10-15 ppm. Aparte de contribuir a un funcionamiento eficaz de los catalizadores y de reducir la emisión de partículas, estas disminuciones adicionales de los niveles de azufre harán posible dar cumplimiento a normas de emisión más exigentes, mediante el uso de los catalizadores DeNOx de la próxima generación, especialmente los sistemas de adsorbentes de NOx. Actualmente éstos son en extremo sensibles al azufre. Una tecnología alternativa de control de emisiones con miras a vehículos más limpios que se ajusta a la normativa Euro V es la reducción catalítica selectiva (RCS). Estos sistemas no son particularmente sensibles a los niveles de azufre en el combustible.

También se sabe que el contenido de azufre incide en el desgaste general y de los depósitos del motor, particularmente en condiciones de baja temperatura y de funcionamiento intermitente. En estas condiciones hay más condensación de la humedad, que se combina con los compuestos de azufre para formar ácidos, lo que ocasiona corrosión y un desgaste excesivo del motor. Por lo general, niveles más bajos de azufre disminuyen el desgaste del motor. Con los estándares de emisión Euro IV+ o equivalentes, el papel del aceite para los motores será igualmente decisivo para asegurar un desempeño sostenido de los motores / dispositivos del tubo de escape. Los niveles bajos de azufre también permiten intervalos más largos de cambio de aceite, reduciendo los costos operacionales.

El combustible diésel tiene propiedades naturales de lubricidad a partir de compuestos que incluyen hidrocarburos más pesados y el azufre orgánico. Las bombas de combustible diésel (especialmente las bombas rotativas de inyección en los vehículos livianos), sin un sistema de lubricación externo, dependen de las propiedades de lubricación del combustible para asegurar un funcionamiento adecuado. El proceso de refinación para eliminar el azufre y los aromáticos del combustible diésel también tiende a reducir los componentes que proveen lubricidad natural. Además de un desgaste excesivo de la bomba y, en algunos casos, de falla del motor, algunas formas de deterioro en el sistema de inyección también podrían afectar el proceso de combustión y, por ende, las emisiones. Existen aditivos para mejorar la lubricidad en combustibles con muy bajo contenido de azufre, y debieran emplearse en cualquier combustible que contenga 500 ppm o menos. 
Los Cuadros $\mathrm{N}^{\circ} 2$ y $\mathrm{N}^{\circ} 3$ presentan un breve resumen del efecto de diversos parámetros del combustible diésel en las emisiones de los vehículos diésel.

En resumen, desde el punto de vista de la tecnología para el control de emisiones, el parámetro diésel más importante es el contenido de azufre en el combustible. Una vez que se incorporen estándares suficientemente rigurosos que exijan catalizadores de oxidación, el contenido de azufre debería reducirse a un máximo de 500 ppm; para los controles más avanzados de NOx y MP, el contenido máximo de azufre debería ser de 10-15 ppm. Si el contenido de azufre sobrepasa estos niveles, no se alcanzará el rendimiento óptimo de los sistemas de control de contaminación y probablemente las emisiones reales superen las normas. Según sea la tecnología escogida por el fabricante, en el caso de los vehículos más limpios, podría producirse un daño permanente si se emplean combustibles con un mayor contenido de azufre.

\section{b) Vehículos y combustibles a gasolina}

La gasolina es una mezcla compleja de hidrocarburos volátiles que se emplea como combustible en motores de combustión interna. Los contaminantes que concitan mayor alarma en el caso de los vehículos que usan gasolina son $\mathrm{CO}, \mathrm{HC}$, NOx, plomo y ciertos hidrocarburos tóxicos tales como el benceno. Cada uno de éstos puede verse afectado por la composición de la gasolina utilizada por el vehículo.

El tratamiento de los gases de escape mediante catalizadores requirió la eliminación del plomo de la gasolina. Este cambio, que comenzó durante la década del 70 y que en la actualidad ha ocurrido en la mayor parte del mundo, ha reducido drásticamente el nivel de plomo presente en el ambiente. Otras propiedades de la gasolina que pueden ajustarse para reducir las emisiones incluyen, aproximadamente en orden de importancia, el nivel de azufre, la presión de vapor, las características de destilación, el contenido de olefina liviana y el contenido de aromáticos ${ }^{11}$.

Los motores modernos a gasolina emplean la inyección computarizada de combustible con control de retroalimentación basado en un

${ }^{11}$ R. F. Sawyer reformuló la gasolina para disminuir las emisiones vehiculares. Véase su libro Twenty-Fourth Symposium (International) on Combustion, 1992, pp. 1423-1432. 
CUADRO N ${ }^{\circ}$ 2: $\quad$ EFECTO DE LAS PROPIEDADES DEL COMBUSTIBLE DIÉSEL EN LOS VEHÍCULOS DIÉSEL LIVIANOS

\begin{tabular}{|c|c|c|c|c|}
\hline $\begin{array}{l}\text { Propiedad del } \\
\text { combustible diésel }\end{array}$ & $\begin{array}{l}\text { Controles } \\
\text { moderados }\end{array}$ & $\begin{array}{l}\text { Controles } \\
\text { fuertes }\end{array}$ & $\begin{array}{l}\text { Controles } \\
\text { avanzados }\end{array}$ & Comentarios \\
\hline Azufre $\uparrow$ & $\begin{array}{l}\mathrm{SO}_{2} \\
\mathrm{MP} \uparrow\end{array}$ & $\begin{array}{c}\text { De } \\
\text { emplearse } \\
\text { cat. de ox., } \\
\mathrm{SO}_{3}, \mathrm{SO}_{2}, \mathrm{MP} \uparrow\end{array}$ & $\begin{array}{c}\text { De } \\
\text { emplearse } \\
\text { filtro, } \\
50 \text { ppm máx., } \\
\text { 10-15 ppm } \\
\text { mejor }\end{array}$ & $\begin{array}{l}\text { De emplearse } \\
\text { adsorbente de } \\
\text { NOx, se } \\
\text { requiere casi } \\
\text { cero de S } \\
\text { (<10 ppm). } \\
\text { Con bajo S, } \\
\text { usar aditivos } \\
\text { de lubricidad. }\end{array}$ \\
\hline Cetano $\uparrow$ & \multicolumn{3}{|c|}{$\begin{array}{l}\text { CO, HC, benceno, 1,3 butadieno, } \\
\text { formaldehído \& acetaldehído } \\
\text { más bajos }\end{array}$} & $\begin{array}{l}\text { Más humo blanco } \\
\text { con combustibles con } \\
\text { bajo índice de cetano. }\end{array}$ \\
\hline Densidad $\downarrow$ & \multicolumn{3}{|c|}{$\begin{array}{l}\text { MP, HC, CO, formaldehído, } \\
\text { acetaldehído \& benceno } \downarrow \text {, NOx } \uparrow\end{array}$} & \\
\hline $\begin{array}{l}\text { Volatilidad (T95 } \\
\left.\text { de } 370 \text { a } 325^{\circ} \mathrm{C}\right)\end{array}$ & \multicolumn{3}{|c|}{$\begin{array}{l}\text { Aumento de NOx, HC, } \\
\text { disminución de MP, CO }\end{array}$} & \\
\hline Poliaromáticos $\downarrow$ & \multicolumn{3}{|c|}{$\begin{array}{l}\text { NOx, MP, formaldehído \& acetaldehído } \downarrow \\
\text { pero HC, benceno \& CO } \uparrow\end{array}$} & $\begin{array}{l}\text { Algunos estudios } \\
\text { demuestran que los } \\
\text { aromáticos totales } \\
\text { son importantes. }\end{array}$ \\
\hline
\end{tabular}

CUADRO No $3: \quad$ EFECTO DE LAS PROPIEDADES DEL COMBUSTIBLE DIÉSEL EN LOS VEHÍCULOS DIÉSEL PESADOS

\begin{tabular}{|c|c|c|c|c|}
\hline $\begin{array}{c}\text { Propiedad del } \\
\text { combustible diésel }\end{array}$ & $\begin{array}{l}\text { Controles } \\
\text { moderados }\end{array}$ & $\begin{array}{l}\text { Controles } \\
\text { fuertes }\end{array}$ & $\begin{array}{l}\text { Controles } \\
\text { avanzados }\end{array}$ & Comentarios \\
\hline Azufre $\uparrow$ & $\mathrm{SO}_{2}, \mathrm{MP} \uparrow$ & $\begin{array}{c}\text { De emplearse } \\
\text { cat. de ox., } \mathrm{SO}_{3} \text {, } \\
\mathrm{SO}_{2}, \mathrm{MP} \uparrow\end{array}$ & $\begin{array}{c}\text { De emplearse } \\
\text { filtro, } 50 \mathrm{ppm} \\
\text { máx., } \\
10-15 \mathrm{ppm} \\
\text { mejor }\end{array}$ & $\begin{array}{l}\text { De emplearse } \\
\text { adsorbente de } \\
\text { NOx, se } \\
\text { requiere casi } \\
\text { cero de azufre } \\
\text { ( }<10 \text { ppm). } \\
\text { Con bajo S, } \\
\text { usar aditivos } \\
\text { de lubricidad. }\end{array}$ \\
\hline Cetano $\uparrow$ & \multicolumn{3}{|c|}{$\begin{array}{c}\text { CO, HC, benceno, } \\
\text { 1,3 butadieno, formaldehído } \\
\& \text { acetaldehído más bajos }\end{array}$} & $\begin{array}{l}\text { Más humo blanco } \\
\text { con combustibles con } \\
\text { bajo índice de cetano. }\end{array}$ \\
\hline Densidad $\downarrow$ & \multicolumn{3}{|c|}{$\mathrm{HC}, \mathrm{CO} \uparrow, \mathrm{NOx} \downarrow$} & \\
\hline $\begin{array}{c}\text { Volatilidad } \\
\text { (T95 de } 370 \text { a } 325{ }^{\circ} \mathrm{C} \text { ) }\end{array}$ & \multicolumn{3}{|c|}{$\begin{array}{l}\text { NOx levemente menores, } \\
\text { pero más HC }\end{array}$} & $\begin{array}{l}\text { Demasiados cortes } \\
\text { pesados aumentan } \\
\text { el humo y MP. }\end{array}$ \\
\hline Poliaromáticos $\downarrow$ & \multicolumn{3}{|c|}{ NOx, MP, HC $\downarrow$} & $\begin{array}{l}\text { Algunos estudios } \\
\text { demuestran que los } \\
\text { aromáticos totales } \\
\text { son importantes. }\end{array}$ \\
\hline
\end{tabular}


sensor de oxígeno para medir con precisión la cantidad y sincronización del combustible que llega al motor. El control de la mezcla en el interior de los cilindros y el uso de ignición de alta energía promueven una combustión casi completa. El catalizador de tres vías hace factible reducir el monóxido de carbono, los hidrocarburos y los óxidos de nitrógeno en más del 90\%. Los diseños para calentamiento rápido minimizan las emisiones de las partidas en frío. Los sistemas de diagnóstico a bordo (DAB) controlan el funcionamiento de los sistemas de emisiones e identifican fallas de los componentes. Una vida útil sobre los $160.000 \mathrm{~km}$ con mínimo mantenimiento es común en muchos países.

\section{Plomo}

A contar de los años veinte, los aditivos de plomo se han mezclado con la gasolina para, en primer lugar, incrementar el octanaje ${ }^{12}$. El plomo no es un componente natural de la gasolina y se añade durante el proceso de refinación, ya sea como tetrametil plomo o tetraetil plomo.

Los vehículos que emplean gasolina con plomo no pueden usar convertidor catalítico y, por consiguiente, sus emisiones contienen niveles más altos de CO, HC y NOx. Además, el mismo plomo es tóxico. Hace mucho tiempo que se ha reconocido el alto riesgo del plomo para la salud. Se absorbe después de inhalarse o ingerirse, y puede producir una amplia gama de efectos biológicos, dependiendo del nivel y duración de la exposición. Los niños, especialmente los menores de cuatro años, son más susceptibles que los adultos a los efectos adversos de la exposición al plomo.

Casi todos los países del mundo han eliminado el uso de la gasolina con plomo; la última estimación es que sólo diecisiete países continúan añadiendo plomo.

\section{Azufre}

El azufre es un componente natural del petróleo crudo. Su proporción en la gasolina refinada depende de la fuente del crudo y el nivel de eliminación de azufre durante el proceso de refinación.

12 Rosner y Markowitz: “A 'Gift of God'?: The Public Health Controversy over Leaded Gasoline During the 1920’s”, 1985. 
El azufre en la gasolina reduce la eficiencia de los catalizadores diseñados para limitar las emisiones de los vehículos y afecta adversamente los sensores de oxígeno. La gasolina con alto contenido de azufre es una barrera para la introducción de nuevas tecnologías de combustión pobre mediante catalizadores DeNOx, en tanto que las gasolinas con bajo contenido de azufre permitirán a las futuras tecnologías vehiculares convencionales desarrollar todos sus potenciales beneficios. Si se bajan los niveles de azufre, las emisiones de los vehículos actuales que vienen con catalizador serán, por lo general, menores.

Las pruebas de laboratorio de los catalizadores han demostrado disminuciones de eficiencia, como consecuencia de mayores niveles de azufre, en una amplia gama de razones aire-combustible. El efecto es mayor, porcentualmente, en los vehículos de bajas emisiones que en los vehículos tradicionales. Los estudios han demostrado que el azufre afecta adversamente los sensores de oxígeno en los gases de escape, hace más lenta la transición de mezcla pobre a rica, incorporando una inclinación espontánea a mezcla rica en la calibración de las emisiones, y puede afectar la vida útil de los sistemas avanzados de diagnóstico a bordo (DAB).

El estudio del Programa Europeo sobre Emisiones, Combustibles y Tecnologías de Motores (EPEFE, por su sigla en inglés) demostró la relación entre niveles reducidos de azufre en la gasolina y disminuciones en las emisiones de los vehículos. Reveló que al disminuir el azufre se reducían las emisiones en los gases de escape de HC, CO y NOx (los efectos generalmente eran lineales con disminuciones de 8 a $10 \%$ cuando el azufre se reducía de $382 \mathrm{ppm}$ a $18 \mathrm{ppm})^{13}$. Los resultados del estudio confirmaron que el azufre en el combustible afecta la eficiencia del catalizador, sobre todo en la función de precalentamiento.

La combustión del azufre produce dióxido de azufre $\left(\mathrm{SO}_{2}\right)$, un irritante ácido que también induce la lluvia ácida y la formación de material particulado sulfatado.

Ciertos aditivos adicionales que se incorporan a la gasolina también pueden afectar las emisiones de los vehículos. Estos aditivos, de composición metálica, formadores de cenizas y mejoradores de octanaje, tales como el metilciclopentadienil manganeso tricarbonil (MMT) y el ferroceno, añadidos a la gasolina aumentarán las emisiones de óxido de

${ }^{13}$ El estudio encontró que los efectos tendían a ser mayores cuando se circulaba a altas velocidades que a bajas velocidades. 
manganeso y óxido de hierro respectivamente en todas las categorías de vehículos. Preocupados por los asuntos de salud, los participantes de un taller convocado por los Comités Científicos de Neurotoxicología y Psicofisiología y Toxicología de Metales de la Comisión Internacional de Salud Ocupacional publicaron recientemente su conclusión de que "[l]a incorporación de compuestos de manganeso orgánico a la gasolina debería detenerse de inmediato en todas las naciones"14. El Instituto de Efectos en la Salud de la OMS había señalado "[q]ue hay abundante evidencia de que (1) en determinadas circunstancias, el manganeso puede acumularse en el cerebro ${ }^{15}$, (2) que la exposición crónica puede producir daño neurotóxico irreversible, (3) que el manganeso puede causar efectos neuroconductuales en dosis relativamente bajas ${ }^{16}$, y (4) que estos efectos siguen a la inhalación de partículas que contienen manganeso".

Los fabricantes de vehículos han manifestado preocupación por la obturación del catalizador y el daño del sensor de oxígeno con el uso de estos aditivos, lo cual ocasionaría mayores emisiones de los vehículos en uso, especialmente a mayor millaje. El efecto parece mayor en los vehículos que se ajustan a normativas estrictas y que emplean sustratos catalizadores de alta densidad celular.

En el Cuadro $\mathrm{N}^{\circ} 4$ se presenta un breve resumen de diversos parámetros de la gasolina en las emisiones vehiculares.

\section{Comentarios finales sobre los vehículos y combustibles}

Una de las lecciones más importantes aprendidas en los cerca de cincuenta años de historia del control de la contaminación vehicular a nivel mundial es que los vehículos y los combustibles deben tratarse

14 Landrigan, P., M. Nordberg, R. Lucchini, G. Nordberg, P. Grandjean, A. Iregren y L. Alessio: "The Declaration of Brescia on Prevention of the Neurotoxicity of Metals”, 2006.

${ }^{15}$ Hauser, R. A., T. A. Zesiewicz, C. Martinez, A. S. Rosemurgy y C. W. Olanow: "Blood Manganese Correlates with Brain Magnetic Resonance Imaging Changes in Patients with Liver Disease”, 1996, pp. 95-98; Lucchini, R., E. Albini, D. Placidi, R. Gasparotti, M. G. Pigozzi, G. Montani y L. Alessio: "Brain Magnetic Resonance Imaging and Manganese Exposure”, 2000, pp. 769-775.

16 Roels, H. A., P. Ghyselen, J. P. Buchet, E. Ceulemans y R. R. Lauwerys: "Assessment of the Permissible Exposure Level to Manganese in Workers Exposed to Manganese Dioxide Dust”, 1992, pp. 25-34. 
CUADRO N 4: $\quad$ EFECTO DE LA COMPOSICIÓN DE LA GASOLINA EN LAS EMISIONES DE VEHÍCULOS LIVIANOS

\begin{tabular}{|c|c|c|c|c|}
\hline Gasolina & $\begin{array}{l}\text { Sin } \\
\text { catalizador }\end{array}$ & $\begin{array}{l}\text { Primeros catalizadores } \\
\text { de tres vías }\end{array}$ & \multicolumn{2}{|c|}{$\begin{array}{l}\text { Catalizadores } \\
\text { más avanzados }\end{array}$} \\
\hline Plomo $\uparrow$ & $\mathrm{Pb}, \mathrm{HC} \uparrow$ & \multicolumn{3}{|c|}{$\begin{array}{l}\text { CO, HC, NOx aumentan drásticamente } \\
\text { a medida que el catalizador se destruye }\end{array}$} \\
\hline $\begin{array}{c}\text { Azufre } \uparrow \\
(50 \text { a } 450 \mathrm{ppm})\end{array}$ & $\mathrm{SO}_{2} \uparrow$ & \multicolumn{3}{|c|}{$\begin{array}{l}\mathrm{CO}, \mathrm{HC}, \mathrm{NOx} \text { aumentan } \sim 15-20 \% \\
\mathrm{SO}_{2} \mathrm{y} \mathrm{SO}_{3} \text { aumentan }\end{array}$} \\
\hline Olefinas $\uparrow$ & \multicolumn{4}{|c|}{$\begin{array}{l}\text { Más 1,3 butadieno, mayor reactividad de HC, más NOx, } \\
\text { pequeño aumento de HC para Euro } 3 \text { y más limpios }\end{array}$} \\
\hline \multirow[t]{2}{*}{ Aromáticos $\uparrow$} & \multicolumn{4}{|c|}{ Más benceno en los gases de escape } \\
\hline & $\begin{array}{l}\text { Potenciales aumentos } \\
\text { de HC, NOx }\end{array}$ & $\begin{array}{l}\mathrm{HC} \uparrow, \mathrm{NOx} \downarrow, \\
\mathrm{CO} \uparrow\end{array}$ & \multicolumn{2}{|c|}{ HC, NOx, CO $\uparrow$} \\
\hline Benceno $\uparrow$ & \multicolumn{4}{|c|}{$\begin{array}{l}\text { Más benceno en los gases de escape } \\
\text { y emisiones evaporativas }\end{array}$} \\
\hline $\begin{array}{c}\text { Etanol } \uparrow \\
\text { hasta } 3,5 \% \mathrm{O}_{2}\end{array}$ & $\begin{array}{l}\text { CO, HC más bajos, } \\
\text { leve aumento de NOx } \\
\text { (cuando el contenido de } \\
\text { oxígeno supera el 2\%), } \\
\text { más aldehídos }\end{array}$ & \multicolumn{3}{|c|}{$\begin{array}{l}\text { Mínimo efecto en los vehículos nuevos } \\
\text { que vienen con sensores de oxígeno, } \\
\text { sistemas adaptativos de aprendizaje }\end{array}$} \\
\hline $\begin{array}{l}\text { MTBE } \uparrow \text { hasta } \\
2,7 \% \mathrm{O}_{2}\end{array}$ & $\begin{array}{l}\text { Menos CO, } \\
\text { HC, más aldehídos }\end{array}$ & \multicolumn{3}{|c|}{$\begin{array}{l}\text { Mínimo efecto en los vehículos } \\
\text { nuevos que vienen con } \\
\text { sensores de oxígeno, } \\
\text { sistemas adaptativos de aprendizaje }\end{array}$} \\
\hline $\begin{array}{l}\text { Propiedades de } \\
\text { destilación } \\
\text { T50, T90个 }\end{array}$ & Probablemente $\mathrm{HC} \uparrow$ & \multicolumn{3}{|c|}{$\mathrm{HC} \uparrow$} \\
\hline $\mathrm{MMT} \uparrow$ & $\begin{array}{l}\text { Más emisiones de } \\
\text { manganeso }\end{array}$ & \multicolumn{2}{|c|}{$\begin{array}{l}\text { Posible } \\
\text { obturación del } \\
\text { catalizador }\end{array}$} & $\begin{array}{c}\text { Probable } \\
\text { obturación del } \\
\text { catalizador }\end{array}$ \\
\hline $\mathrm{PVR} \uparrow$ & \multicolumn{4}{|c|}{ Más emisiones evaporativas de HC } \\
\hline $\begin{array}{c}\text { Aditivos para el } \\
\text { control de } \\
\text { depósitos } \uparrow\end{array}$ & \multicolumn{4}{|c|}{$\begin{array}{l}\text { Potenciales beneficios para las emisiones } \\
\text { de HC y NOx }\end{array}$} \\
\hline
\end{tabular}

Fuente: Elaboración propia del autor. 
como un sistema. Las mejoras en los vehículos y los combustibles deben ir de la mano si se espera avanzar en la disminución de la contaminación producida por los vehículos. Un programa focalizado únicamente en los vehículos está condenado al fracaso; de igual manera, tampoco tendrá éxito un programa diseñado para mejorar solamente la calidad del combustible.

Tal como se analizó anteriormente, los combustibles diésel reformulados pueden reducir las emisiones particuladas de todos los vehículos diésel. Los combustibles con índices especialmente bajos de azufre reducen el aporte de sulfatos. Ciertas tecnologías de post-tratamiento son especialmente sensibles al contenido de azufre en el combustible. Por consiguiente, en caso de requerirse un control muy estricto de NOx y MP, será preciso reducir a 50 ppm o menos los niveles de azufre e incorporar los estándares Euro IV para vehículos. Los estándares Euro V o los estándares norteamericanos Tier 2 incluyen un límite para el azufre en el combustible de 10-15 ppm. Ya están disponibles las tecnologías para alcanzar estos niveles y están apareciendo tecnologías aún más avanzadas para los vehículos nuevos.

Con respecto a los vehículos que emplean gasolina, el uso del tratamiento de los gases de escape mediante catalizador requiere que se elimine el plomo de la gasolina. Este cambio, que se ha producido en casi todo el mundo, ha ocasionado una drástica reducción de los niveles de plomo en el ambiente. Otras propiedades de la gasolina que pueden ajustarse para disminuir las emisiones incluyen, aproximadamente en orden de importancia, nivel de azufre, presión de vapor, características de destilación, contenido de olefinas livianas y contenido de aromáticos ${ }^{17}$. Está apareciendo la tecnología para los catalizadores destinados a los vehículos de dos y tres ruedas, motivo por el cual también será importante una gasolina sin plomo y con un contenido de azufre menor para estos vehículos.

\section{Normas estrictas sobre emisiones vehiculares}

Los tres programas mundiales predominantes corresponden a EE.UU. (incluida California), la Unión Europea (UE) y Japón. Muchos países han adoptado los estándares y procedimientos de pruebas euro-

17 Sawyer, R. F.: Twenty-Fourth Symposium (International) on Combustion, 1992, pp. 1423-1432. 
peos y norteamericanos, o alguna mezcla de ellos. Por ejemplo, China e India han adoptado los estándares de la UE para la mayoría de las categorías de vehículos, aunque con varios años de retraso en su implementación respecto de la UE. En lo que atañe a los vehículos de pasajeros, cerca del 60\% del parque mundial está siguiendo el esquema regulatorio de la UE, y casi el 30\% sigue la senda de los EE.UU. No es de sorprender que la gran mayoría, cerca del 90\% de los autos diésel, siga el camino adoptado por la UE. En relación con los camiones livianos, cerca del $60 \%$ sigue los estándares norteamericanos, en tanto que sobre el $70 \%$ de los camiones pesados sigue los estándares sobre emisiones de la UE. Ningún país fuera de Japón exige los estándares japoneses.

Es importante destacar dos puntos importantes:

1. Los estándares adoptados por EE.UU. y la UE determinarán los tipos de tecnologías y controles de contaminación que empleará la mayoría de los vehículos livianos y pesados alrededor del mundo, de tal modo que es importante que sus estándares sean lo suficientemente estrictos como para abordar los problemas ambientales para los cuales están diseñados, y

2. Si bien la brecha de tiempo se está acortando, muchos países están atrasados en relación con EE.UU. y la UE en cinco o más años en la implementación de los estándares.

Las tecnologías se encuentran disponibles en el mercado o están apareciendo rápidamente, y éstas, en combinación con los combustibles limpios que ya analizamos, pueden reducir, por kilómetro conducido, las emisiones vehiculares de CO, HC, NOx y MP y otros contaminantes a una fracción muy pequeña, en comparación con las de los vehículos sin controlar, y el principal desafío en la actualidad es que estas tecnologías se adopten a nivel mundial.

En relación con los vehículos y motores pesados, EE.UU. y Japón están en vías de introducir requisitos muy rigurosos para NOx y MP antes que finalice la presente década. En el caso de Japón, los requisitos incluyen uno obligatorio para el NO y un así llamado valor de desafío que solamente corresponde a la tercera parte del requisito obligatorio. Si el valor de desafío se torna obligatorio, los requisitos japoneses serán muy similares a los norteamericanos para el año 2010. 
CUADRO N 5: $\quad$ NORMAS EURO III PARA MOTOCICLETAS (2006)

\begin{tabular}{lccc}
\hline HC $(\mathrm{g} / \mathrm{km})$ & NOx $(\mathrm{g} / \mathrm{km})$ & $\mathrm{CO}(\mathrm{g} / \mathrm{km})$ & Vida útil $(\mathrm{km})$ \\
\hline 0,8 & 0,15 & 2,0 & 30.000 \\
\hline
\end{tabular}

Con respecto a los requisitos Euro VI para vehículos pesados, la Comisión Europea emitió una propuesta en diciembre de 2007, cuyo objetivo es que sean casi equivalentes a los límites fijados por EE.UU. para 2010. El Parlamento Europeo recientemente aprobó la propuesta con apenas unos pocos cambios.

En 2006, la Unión Europea introdujo los estándares Euro III para motocicletas (véase el Cuadro $\mathrm{N}^{\circ}$ ), que son casi equivalentes a los estándares para autos nuevos aplicados en la UE el año 2000, y estas exigencias han suscitado el interés de otros países. Tanto Taiwán (2007) como China (2008) han anunciado su intención de adoptar los requisitos de la UE con leves modificaciones.

\section{Cambio climático global}

Con respecto a los GEI (gases de efecto invernadero), el pronóstico es menos promisorio. Las emisiones equivalentes de $\mathrm{CO}_{2}$ del sector transporte crecieron significativamente en los países desarrollados (Anexo 1 del Protocolo de Kioto) entre 1990 y 2004 ${ }^{18}$; de hecho, el crecimiento del sector, $24 \%$, fue lejos el mayor de todos los sectores durante este período.

\section{Estrategias para reducir los gases de efecto invernadero y la contaminación del aire}

A continuación se presentan tres enfoques sobre una base tecnológica para reducir los GEI en el sector transporte:

${ }^{18}$ United Nations Framework Convention on Climate Change (UNFCCC): "National Greenhouse Gas Inventory Data for the Period 1990-2004, and Status of Reporting”, 19 de octubre, 2006. 
- $\quad$ Fijación de normas obligatorias o voluntarias sobre emisiones o estándares de eficiencia del combustible;

- Conversión a combustibles de bajo carbono y tecnologías vehiculares avanzadas; $y$

- $\quad$ Reducción en el uso de vehículos motorizados.

\section{a) Normas para vehículos}

El estado de California ha fijado estándares en relación con los gases de efecto invernadero y está a la espera de una autorización de la Agencia de Protección Ambiental de Estados Unidos (EPA, por su sigla en inglés) para su implementación ${ }^{19}$. En Estados Unidos, a nivel local, las normas obligatorias de ahorro de combustible (CAFE, Corporate Average Fuel Economy) han existido desde mediados de los años setenta, pero no se habían hecho ajustes significativos en más de veinte años hasta que el Congreso decretó un mayor control en 2007; estas exigencias debieran conducir a una disminución de las emisiones de dióxido de carbono, pero no consideran otras emisiones de efecto invernadero. La Unión Europea, por su parte, ha negociado un acuerdo voluntario con la industria automotriz europea para alcanzar metas respecto al dióxido de carbono ${ }^{20}$. Este acuerdo fracasó a comienzos de 2007 cuando quedó claro que la meta de $140 \mathrm{~g} / \mathrm{km}$ prevista para el año 2008 no se cumpliría. Por consiguiente, la UE impondrá un límite obligatorio de $130 \mathrm{~g} / \mathrm{km}$ que se introducirá en forma progresiva entre 2012 y 2015, y muy probablemente ajustará los límites a alrededor de $95 \mathrm{~g} / \mathrm{km}$ en 2020.

Para estimular el progreso de la industria, el enfoque de Japón también se ha centrado en que el combustible utilizado sea, en cada momento, el mejor de su clase.

En el Gráfico $\mathrm{N}^{0} 3$ se presenta un resumen de las exigencias proyectadas o adoptadas ${ }^{21}$.

19 The International Council on Clean Transportation: "Passenger Vehicle $\mathrm{CO}_{2}$ and Fuel Economy Standards: A Global Update”, 2008.

${ }^{20}$ Se lograron acuerdos similares con los fabricantes japoneses y coreanos.

${ }^{21}$ ICCT (The International Council on Clean Transportation): "Passenger Vehicle $\mathrm{CO}_{2}$ and Fuel Economy Standards: A Global Update”, agosto de 2008. 
GRÁFICO No 3: PROYECCIÓN DE EMISIONES DE GEI PARA LOS VEHÍCULOS NUEVOS DE PASAJEROS POR PAÍS/REGIÓN

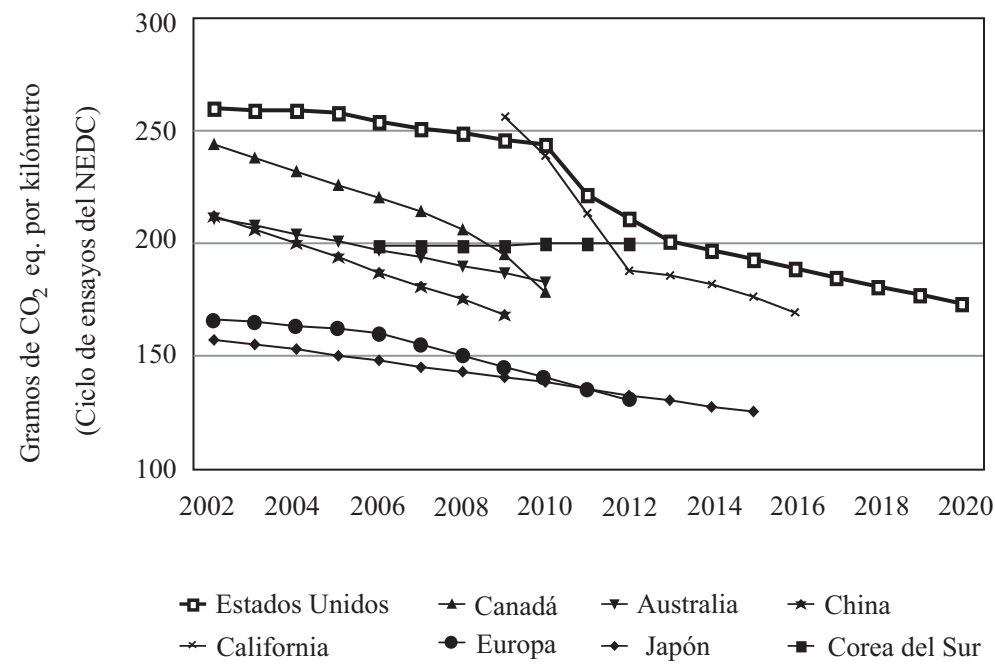

Fuente: ICCT (The International Council on Clean Transportation), agosto de 2008.

\section{b) Combustibles bajos en carbono}

El objetivo de los estándares para combustibles bajos en carbono (LCFS, por sus siglas en inglés) es promover, precisamente, la inversión y el uso de combustibles bajos en carbono (por ejemplo, etanol de maíz y biodiésel sustentables, gas natural comprimido, electrones/hidrógeno renovables) y atenuar la demanda por combustibles altos en carbono (por ejemplo, las arenas asfálticas canadienses, el petróleo de esquistos bituminosos venezolano y el carbón líquido de Estados Unidos).

Brasil fue el primer país en realizar una conversión significativa a combustibles renovables bajos en carbono, pero muchos países están adoptando enfoques similares con variados resultados. California propuso recientemente exigencias para combustibles en base a carbono y la UE está adoptando estándares de combustibles bajos en carbono ${ }^{22}$. Sin embargo, para lograr beneficios globales importantes de los combustibles

${ }^{22}$ Farrell, A., D. Sperling et al.: “A Low Carbon Fuel Standard for California, Part 1: Technical Analysis”, mayo 29, 2007. 
bajos en carbono se hace cada vez más evidente la necesidad de un análisis completo del ciclo de vida que considere los efectos indirectos del uso del suelo. Cuando dichos factores se toman en consideración, queda claro que la conversión a combustibles bajos en carbono que realmente logren beneficios significativos es algo difícil de lograr.

El actual estándar de combustibles renovables de Estados Unidos (RFS, por sus siglas en inglés) es un paso hacia los estándares de combustibles bajos en carbono al requerir normas para el ciclo de vida de los gases de efecto invernadero de tres categorías de biocombustibles: biocombustibles renovables básicos, 20\% bajo la gasolina; biocombustibles avanzados, 50\% de mejoría; y los biocombustibles celulósicos, $60 \%$ de mejoría. Sin embargo, los estándares para combustibles renovables sólo se aplican a los biocombustibles y, por consiguiente, no disminuyen la demanda por combustibles altos en carbono (por ejemplo, las arenas asfálticas, el carbón líquido).

En California, un proyecto de regulación para los combustibles bajos en carbono, el cual requiere que los proveedores de combustible reduzcan la intensidad del carbono en todos los combustibles empleados por el sector transporte en un 10\% para el año 2020, fue presentado en octubre de 2008. California espera tener lista la regulación en marzo de 2009. Los servicios eléctricos pueden optar por el programa y generar créditos si venden "electrones renovables" a híbridos enchufables, o vehículos totalmente eléctricos. Las emisiones de gases de efecto invernadero que obedecen a cambios directos e indirectos del uso del suelo están incluidas en la estimación de los efectos de los GEI a lo largo del ciclo de vida del combustible.

El Parlamento Europeo está debatiendo estándares para combustibles bajos en carbono similares a los de California. La nueva ley requerirá que los proveedores de combustible reduzcan las emisiones de los gases de efecto invernadero durante el ciclo de vida de los combustibles para vehículos en un 6\% entre el 2010 y el 2020 (metas intermedias: 2\% al 31 de diciembre de 2014 y $4 \%$ al 31 de diciembre de 2017). Las reducciones provendrán de mejoras en la eficiencia de la producción y por la conversión a combustibles más limpios, tales como los biocombustibles. Los criterios de sustentabilidad de los biocombustibles se incorporarán a la nueva ley una vez que se llegue a acuerdo sobre ellos en negociaciones separadas para una nueva directiva sobre energía renovable. 
Con ello la UE ha enviado una señal clara de que su mercado no está abierto a los petróleos marginales intensivos en carbono, tales como las arenas asfálticas o el carbón líquido.

Aún quedan varias interrogantes técnicas clave en relación con los combustibles bajos en carbono, tales como:

- La incertidumbre acerca de las emisiones de gases de efecto invernadero durante el ciclo de vida del etanol de maíz estadounidense (el rango se encuentra entre un promedio de mejoría del $25 \%$ con respecto a la gasolina y el doble de las emisiones de gases de efecto invernadero a lo largo del ciclo de vida de la gasolina, lo que hace que el etanol de maíz sea peor que las arenas asfálticas y el carbón líquido).

- Si la biomasa para combustible sólo puede producirse sustentablemente cuando crece en tierra degradada o es producida a partir de productos de desecho (por ejemplo, residuos de maíz, desechos del bosque), ¿cuáles son, entonces, las limitaciones prácticas para un abastecimiento global de biocombustibles sustentables?

- ¿ ¿Qué tanto del reciente aumento del precio mundial del maíz y de otros granos es atribuible a las disposiciones sobre biocombustibles en Estados Unidos y otros países?

- ¿ ¿Cuáles son las perspectivas de los híbridos enchufables y la capacidad de los servicios eléctricos para proveer suficientes electrones renovables a este nuevo mercado?

\section{Tecnologías vehiculares avanzadas}

California exige y Japón promueve fuertemente tecnologías vehiculares avanzadas, que incluyen los autos eléctricos a batería, los híbridos, los híbridos enchufables y las baterías de combustible, con lo cual se están obteniendo logros significativos ${ }^{23}$; sobre 300.000 vehículos híbridos se vendieron en el mundo el año 2006.

La situación y las perspectivas de los vehículos de emisión cero (ZEV: zero emission vehicles), así como los vehículos de tecnología

${ }^{23}$ Walsh, M. P. et al.: "Status and Prospects for Zero Emissions Vehicle Technology”, 2007, preparado para el State of California Air Resources Board, Sacramento, California, abril 13, 2007. 
avanzada (ATV: advanced technology vehicles) que podrían tener beneficios sinergéticos que respalden la introducción de ZEV se resumen a continuación.

\section{a) Vehículo eléctrico a batería de alto rendimiento (FPBEV: full performance battery electric vehicle)}

Los vehículos eléctricos a batería de alto rendimiento son vehículos a batería (BEV, por sus siglas en inglés) plenamente capaces de circular en carreteras urbanas y suburbanas de alta velocidad. Pese a significativos avances tecnológicos, los esfuerzos previos para introducir los FPBEV no fueron exitosos; las grandes baterías requeridas para proveer el rango necesario de desplazamiento aún son muy caras.

Los altos precios de los combustibles y las condiciones más favorables de conducción en Japón y Europa reducen las barreras para los FPBEV, y como resultado de ello algunos fabricantes están desarrollando pequeños FPBEV con baterías de ion litio para estos mercados.

Debido al alto costo de las baterías, el cual no se recupera con el ahorro por concepto de combustible, y a una aceptación limitada por cuestiones de alcance y tiempo de recarga, es improbable que los FPBEV se conviertan en el futuro inmediato en un mercado masivo de vehículos de emisión cero (ZEV).

\section{b) Vehículo eléctrico de ciudad (CEV: city electric vehicle)}

Los vehículos eléctricos de ciudad, con aceleración y velocidad máxima limitadas (a saber: 50/60 mph), y por consiguiente no aptos para circular en carreteras urbanas y suburbanas de alta velocidad, permiten el uso de una batería de menor tamaño y de un sistema eléctrico de menor potencia, de tal modo que el vehículo puede fabricarse a un menor costo y así estar más al alcance de los usuarios.

Los CEV pueden convertirse en los futuros ZEV de consumo masivo en Japón y Europa.

\section{c) Vehículo eléctrico de vecindario (NEV: neighborhood electric vehicle)}

Los vehículos eléctricos de vecindario, capaces de alcanzar velocidades máximas entre 20 y 25 mph, parecen tener éxito comercial- 
mente hablando, pero tienen un bajo potencial de volumen debido a su limitada aplicabilidad. Igualmente, dado que emplean una tecnología muy simple, tienen muy poca sinergia con los BEV más grandes.

\section{d) Vehículo eléctrico híbrido (HEV: hybrid electric vehicle)}

Los HEV son atractivos para clientes que deseen pagar un sobreprecio. Si bien los productores están bajando los costos de los componentes y sistemas eléctricos, el elevado costo de fabricación todavía es un problema. Sin embargo, los fabricantes están incorporando nuevos elementos, pese al tema del costo, principalmente por motivos competitivos. En general, los volúmenes de venta de los HEV caen y suben junto con el precio de la gasolina.

Los HEV están dando un mayor respaldo a los futuros ZEV de consumo masivo al estimular avances en los sistemas eléctricos, accesorios eléctricos y tecnologías de batería. Asimismo, está aumentando el interés de los clientes por la electropropulsión y sus beneficios asociados.

\section{e) Vehículo eléctrico híbrido enchufable (PHEV: plug-in hybrid electric vehicle)}

Los PHEV prometen diversos beneficios a los usuarios y a la sociedad. La capacidad relativamente pequeña de la batería puede usarse a diario en forma completa, permitiendo que el cliente, de este modo, obtenga un máximo de ahorro en combustible, en recompensa por el sobreprecio inicial pagado por el vehículo.

Recientemente, algunos fabricantes se han interesado en los PHEV, los cuales están atrayendo la atención de los medios y generando altas expectativas en los consumidores. La gama de los vehículos totalmente eléctricos (AER: all electric range) podría tener un efecto importante en el costo de fabricación, así como en requerimientos de inversión de capital si fuesen necesarios sistemas únicos y más poderosos de electropropulsión y, por lo tanto, los AER podrían tener un efecto significativo en el temprano éxito de la tecnología.

Es probable que los PHEV estén disponibles en 2010-2011. Al estimular el desarrollo de baterías de energía y contribuir a que los consumidores acepten la tecnología enchufable, los PHEV podrían promover la producción de futuros BEV en forma masiva. 


\section{f) Vehículo eléctrico con célula de combustible (FCEV: fuel cell electric vehicle)}

Varias empresas que están llevando a cabo ingentes esfuerzos en investigación y desarrollo consideran que los FCEV son la solución máxima. Sin embargo, sigue siendo muy difícil alcanzar simultáneamente los objetivos de rendimiento, vida útil y costo con los FCEV.

Los FCEV híbridos enchufables de serie que operan en régimen tienen el potencial de alcanzar al mismo tiempo los objetivos de rendimiento, vida útil y costo.

Con la tasa de éxito pasada y los recursos intelectuales y financieros masivos que se han asignado a esta tecnología, los FCEV continúan siendo un candidato prometedor para un verdadero mercado masivo de ZEV en el futuro.

\section{g) Conclusiones generales: Las perspectivas de los ZEV}

Los PHEV (vehículos eléctricos híbridos enchufables) con capacidad moderada de almacenamiento derivarán de los HEV y probablemente proliferarán rápidamente, promoviendo el desarrollo y un menor costo de las baterías de energía, orientándose al mismo tiempo a PHEV comercialmente viables y, en el más largo plazo, a los FPBEV (vehículos eléctricos a batería de alto rendimiento). Mientras los PHEV seguirán presentando un rápido crecimiento, puesto que no tienen limitaciones funcionales, los FPBEV crecerán de manera más lenta debido a su limitado alcance y largo tiempo de recarga. Los NEV (vehículos eléctricos de vecindario) son comercialmente viables en la actualidad y continuarán creciendo, aunque en forma más lenta debido a su limitada funcionalidad. Los CEV (vehículos eléctricos de ciudad) se volverán comercialmente viables en Japón y Europa en un futuro no muy lejano. El crecimiento de los CEV será lento debido a las limitaciones funcionales de los BEV en general, y a las limitaciones específicas de los CEV, en especial para circular en carreteras urbanas. El intenso esfuerzo que se está haciendo en los FCEV dará como resultado vehículos técnicamente capaces para 2015-2020, pero una comercialización exitosa dependerá de si se logran los desafiantes objetivos de costo y la disponibilidad de una adecuada infraestructura de hidrógeno. Si ello ocurre, es probable que los FCEV crezcan rápidamente. 


\section{REFERENCIAS BIBLIOGRÁFICAS}

Auto/Oil Air Quality Improvement Research Program: "Final Report”. Enero 1997.

Farrell, A. y D. y Sperling (directores del proyecto) et al.: "A Low Carbon Fuel Standard for California, Part 1: Technical Analysis”, 29 de mayo, 2007.

Hauser, R. A., T. A. Zesiewicz, C. Martinez, A. S. Rosemurgy y C. W. Olanow: "Blood Manganese Correlates with Brain Magnetic Resonance Imaging Changes in Patients with Liver Disease”. En Canadian Journal of Neurological Science, 23 (2) (1996).

Honda Motor Company: “World Motorcycle Facts and Figures”, 2004.

ICCT (The International Council on Clean Transportation): "Passanger Vehicle $\mathrm{CO}_{2}$, and Fuel Economy Standars: A Global Update”. Agosto de 2008.

Landrigan, P., M. Nordberg, R. Lucchini, G. Nordberg, P. Grandjean, A. Iregren y L. Alessio: "The Declaration of Brescia on Prevention of the Neurotoxicity of Metals”. En American Journal of Industrial Medicine, 2006.

Lucchini, R., E. Albini, D. Placidi, R. Gasparotti, M. G. Pigozzi, G. Montani y L. Alessio: "Brain Magnetic Resonance Imaging and Manganese Exposure”. En Neurotoxicology, 21(5) (2000).

Panel Intergubernamental sobre Cambio Climático FALTAN nombres de documentos (1990 y 1996) aludidos en Cuadro 1

Ramanathan, V. y G. Carmichael: “Global and Regional Climate Changes Due to Black Carbon”. En Nature Geoscience, Vol. 1 (abril 2008). Www.nature.com/ naturegeoscience.

Roels, H. A., P. Ghyselen, J. P. Buchet, E. Ceulemans y R. R. Lauwerys: “Assessment of the Permissible Exposure Level to Manganese in Workers Exposed to Manganese Dioxide Dust”. En British Journal of Industrial Medicine, 49(1) (1992).

Rosner, David y Gerald E. Markowitz: “A 'Gift of God’?: The Public Health Controversy over Leaded Gasoline During the 1920's”. En American Journal of Public Health, 1985.

Sawyer, R. F.: Twenty-Fourth Symposium (International) on Combustion. Pittsburgh, Pennsylvania: The Combustion Institute, 1992.

United Nations Framework Convention on Climate Change (UNFCCC): "National Greenhouse Gas Inventory Data for the Period 1990-2004, and Status of Reporting”, 19 de octubre de 2006.

Walsh, M. P. (presidente), F. R. Kalhammer, B. M. Kopf, D. H. Swan y V. P. Roan: "Status and Prospects for Zero Emissions Vehicle Technology". Informe para el ARB Independent Expert Panel 2007, preparado por el State of California Air Resources Board del estado de California, Sacramento, California, 13 de abril, 2007.

Wards Communications Inc.: Ward's Motor Vehicle Data, 2006.

World Business Council on Sustainable Development: "Mobility 2030: Meeting the Challenges to Sustainability”. En The Sustainable Mobility Project, Full Report. 2004. 